\title{
The role of components of the chromatin modification machinery in carcinogenesis of clear cell carcinoma of the ovary (Review)
}

\author{
HIROSHI SHIGETOMI, AKIRA OONOGI, TAIHEI TSUNEMI, YASUHITO TANASE, YOSHIHIKO YAMADA, \\ HIROTAKA KAJIHARA, YORIKO YOSHIZAWA, NAOTO FURUKAWA, SHOJI HARUTA, \\ SHOZO YOSHIDA, TOSHIYUKI SADO, HIDEKAZU OI and HIROSHI KOBAYASHI
}

Department of Obstetrics and Gynecology, Nara Medical University, Nara, Japan

Received January 21, 2011; Accepted April 27, 2011

DOI: $10.3892 / \mathrm{ol} .2011 .316$

\begin{abstract}
Recent data have provided information regarding the profiles of clear cell carcinoma of the ovary (CCC) with adenine-thymine rich interactive domain 1A (ARID1A) mutations. The purpose of this review was to summarize current knowledge regarding the molecular mechanisms involved in CCC tumorigenesis and to describe the central role played by the aberrant chromatin remodeling. The present article reviews the English-language literature for biochemical studies on the ARID1A mutation and chromatin remodeling in CCC. ARID1A is responsible for directing the SWI/SNF complex to target promoters and regulates the transcription of certain genes by altering the chromatin structure around those genes. The mutation spectrum of ARID1A was enriched for $\mathrm{C}$ to $\mathrm{T}$ transitions. CCC and clear cell renal cell carcinoma (ccRCC) resemble each other pathogenetically. Dysfunction of the ARID1A protein, which occurs with VHL mutations in ccRCC, is responsible for loss of the assembly of the ARID1Amediated histone H2B complex. Therefore, ARID1A acts as a chromatin remodeling modifier, which stimulates cell signaling that can lead to cell cycle arrest and cell death in the event of DNA damage. The dysfunction of ARID1A may result in susceptibility to $\mathrm{CCC}$ carcinogenesis through a defect in the repair or replication of damaged DNA.
\end{abstract}

\section{Contents}

1. Introduction

2. Materials and methods

3. Article selection, data extraction and assessment

4. Chromatin remodeling complex in cancers

5. Characteristics of SWI/SNF subunit inactivation in CCC

Correspondence to: Dr Hiroshi Kobayashi, Department of Obstetrics and Gynecology, Nara Medical University, 840 Shijo-cho, Kashihara 634-8522, Japan

E-mail: hirokoba@naramed-u.ac.jp

Key words: adenine-thymine rich interactive domain 1A, chromatin, clear cell carcinoma of the ovary
6. A marked resemblance between CCC and ccRCC

7. Conclusions

\section{Introduction}

Epithelial ovarian cancer (EOC) is the most lethal gynecologic malignancy worldwide. Epidemiology calculations of lifetime risk for EOC are that 1 in 55 women is likely to develop EOC during their lifetime (1). Since EOC is more likely to be advanced stage with unfavorable tumor biology, there are serious limitations to the surgical and oncological treatment available. Therefore, it is crucial to determine the earliest possible diagnosis. Early diagnosis and surgical resection offer patients the best opportunity of excellent long-term survival. On the other hand, patients who either present with metastatic disease or develop distant relapse within 6 months after surgery and chemotherapy have a poor prognosis. Among EOC, clear cell carcinomas of the ovary (CCC) are frequently characterized by chemoresistance and recurrence, resulting in a poor prognosis (2). Since CCC are resistant to conventional cytotoxic (platinum plus taxan-based) chemotherapy, the prognosis is mostly poor (3). In the USA, the incidence of CCC is $5 \%$ of all EOC, but the incidence in Japan is reported to be over $20 \%$ of all EOC. Thus, Japanese oncologists have focused on investigating the molecular pathogenesis and treatment strategies of CCC.

At present, little is known about the molecular genetic mechanisms that are involved in CCC tumorigenesis. Accumulated somatic mutations found in a subset of cancer genes are frequently noted. Such mutations include insertions/deletions (indels) and base substitutions that act as the "driver mutations' in oncogenesis. These mutations result in the activation of proto-oncogenes (gain of function) or the inhibition of tumor suppressor genes (loss of function) during the process of carcinogenesis. Screening for regions with loss of heterozygosity ( $\mathrm{LOH})$ in tumors is widely used to search for novel tumor suppressor genes (4).

Endometriosis has been suggested to increase the risk of developing EOC (5). Compared to the serous surface epithelial subtype (serous adenocarcinoma, SAC), which comprises the majority of EOC, some types such as CCC and endometrioid adenocarcinoma (EAC) have been associated with endometriosis (6). CCC are most frequently associated with ovarian 
and/or pelvic endometriosis in Japan (5). Even in the case of endometriosis, $\mathrm{LOH}$ at $1,5,9,10,11,17$ and $22 \mathrm{q}$ is especially common (7). In total, approximately $30 \%$ of cases exhibited $\mathrm{LOH}$ at one or more of these loci. These studies support the hypothesis that tumor suppressor gene inactivation plays a role in the development of at least one subset of cases $(4,7)$. $\mathrm{LOH}$ studies have shown the involvement of specific chromosomal regions $(5,6,9,10,11,17$ and $22 \mathrm{q})$ in $\mathrm{CCC}(8)$. $\mathrm{LOH}$ of these chromosomes is frequent not only in endometriosis but also in $\mathrm{CCC}$, suggesting that one or more common tumor suppressor genes are present in these regions.

The purpose of this review is to summarize the current knowledge on the molecular mechanisms involved in CCC tumorigenesis and to provide the central role played by aberrant chromatin remodeling. We specifically summarize what is currently known about the salient features of members of the ARID protein family and focus on recent developments in characterizing the functional links of carcinogenesis between $\mathrm{CCC}$ and clear cell renal cell carcinoma (ccRCC).

\section{Materials and methods}

The present article reviews the English-language literature for chromatin remodeling studies on CCC. We searched PubMed electronic databases over a 20-year period (1990-2010), combining the keywords 'ARID1A', 'SWI/SNF', 'chromatin remodeling', 'p53', 'pRb', 'cell cycle', 'check point' with 'endometriosis', 'ovarian cancer', 'endometriosis-associated ovarian cancer (EAOC)', 'clear cell carcinoma of the ovary', or 'clear cell renal cell carcinoma'. Various recent studies are discussed in the context of the pathogenesis of CCC. Additionally, references in each article were searched to identify potentially missed studies for a 10 -year period. In the present review, we evaluate promising molecular candidates for the development of CCC.

\section{Article selection, data extraction and assessment}

Although the main focus of the present review was the regulation of ARID1A mutations obtained from human cancer samples, in vitro studies were included in the knowledge base. Animal models performed to support human data were also included. Initially, 37 potentially relevant studies were identified by screening electronic databases. Additionally, 49 peerreviewed journal articles were identified from references.

\section{Chromatin remodeling complex in cancers}

Chromatin regulates transcriptional processes through coordinated covalent modifications of DNA and its associated nucleosomal histones (9). Such modifications include acetylation, methylation and ubiquitination. Post-translational modifications of histones play critical roles in chromosome dynamics. Epigenetic mechanisms underlying the modification of chromatin structure rely on the activity of complexes that control the accessibility of DNA sequences to transcription factors, thereby determining its different functional states (9). Chromatin remodeling complexes are master regulators of transcription factor action and enable gene transcription by aiding in the coordination of the binding of transcrip- tion factors to promoters and enhancers. These complexes are involved in various processes that require alteration of chromatin structure including DNA repair, DNA synthesis, mitosis and genomic stability (10). Mounting evidence shows that alterations in the subunits of these complexes play a significant role in human disease, including cancer (10). Core components of the chromatin remodeling complexes are potent tumor suppressors that are specifically inactivated in cancers. Alterations of the components have been reported in a variety of cancer types, including central nervous system, head and neck, leukemia, breast, lung, neuroblastoma, renal, skin, gastric, colon, cervical and prostate cancer (10). Results of various studies have also shed light on the mechanistic basis of the action by demonstrating that these components regulate the cell cycle to prevent oncogenic transformation.

\section{Characteristics of SWI/SNF subunit inactivation in CCC}

SWI/SNF. The chromatin remodeling complexes include SWI/ SNF, ISWI (Imitation SWI), CHD/Mi-2 (chromodomain helicase DNA binding protein), and INO80 (SNF2 family helicase). Of these complexes, SWI/SNF is the most studied, consisting of an evolutionarily conserved protein complex from yeast to humans. First, SWI/SNF is a multimeric complex of proteins of variable composition, including ATPase, core and accessory proteins. The functions and components of the SWI/SNF complex have been thoroughly reviewed elsewhere (10). This complex constitutes SMARCs (SWI/SNF-related, matrix-associated, actin-dependent regulators of chromatin; SMARCA/BRGI subunit or SMARCA2/BRM subunit) and the BAF (BRM- or BRG1-associated factors) complex, which comprises 10-12 protein subunits (10-12). The role of this complex may be to protect cells against DNA damage by ensuring DNA repair through cell cycle arrest and apoptosis. Key observations link the SWI/SNF complex with cancer. The complex is associated with multiple cancer-related pathways, and various components of the SWI/SNF complex act as bona fide tumor suppressors (13). Furthermore, cancerrelated proteins such as p21 (also known as p21WAF1/Cip1 and CDKN1A, cyclin-dependent kinase inhibitor 1A), BRCA1 (breast cancer 1, early onset), LKB1 (also known as STK11, serine/threonine kinase 11), SMADs, FOS (FBJ murine osteosarcoma viral oncogene homolog), MYC (v-myc myelocytomatosis viral oncogene homolog) and FANCA (Fanconi anemia, complementation group A) have been associated with certain components of the SWI/SNF complexes. A growing body of evidence has demonstrated that these factors play a critical role in several steps of carcinogenesis, particularly in alterations in the cell cycle checkpoint machinery. p21WAF1/ Cip1 mediates the p53-dependent cell cycle G1 phase arrest in response to a variety of stress stimuli (14). Epigenetic inactivation of BRCA1 impairs the machinery involved in maintaining genomic integrity and stability, and also acts as a bona fide tumor suppressor (15). LKB1/STK11 plays a role in apoptosis and cell cycle arrest, both of which may require the tumor suppressor action of this kinase, whose mutations occur in approximately $50 \%$ of lung cancers (16). LKB1 tumor suppressor enzyme is vulnerable to inactivation by redoxactive species. Other genes such as SMADs, FOS, MYC and FANCA have been shown to play a role as regulators of 
cell cycle progression, apoptosis and cellular transformation (17-19)

BRG1 (Brahma/SWI2-related gene 1). BRG1 (Brahma/ SWI2-related gene 1) is a central component of the SWI/ SNF chromatin-remodeling complex that features an ATPase activity (13). The role of BRG1 is to arrest the cell cycle. Loss of BRG1 has been associated with cancer development. BRG1 deficiency is associated with a subset of lung, breast, prostate and pancreatic cancers (13).

SNF5/INI1. The clearest functional link between the SWI/ SNF complex and cancer is evident from the subunit SNF5/ INI1 (also known as SMARCAB1 or BAF47). SNF5 is a core protein of the SWI/SNF complex. SNF5 leads to a G1 cell cycle arrest associated with an increase in p16INK4a (also known as CDKN2A, cyclin-dependent kinase inhibitor 2A), E2F and cyclin D to prevent oncogenic transformation (20). Importantly, the E2F transcription factor plays a role in cell cycle control and is intimately regulated by $\mathrm{RB}$ (retinoblastoma tumor suppressor gene). Since SNF5 is a potent tumor suppressor, loss of SNF5 can lead to aberrant cell cycle activity and subsequent tumor formation. In particular, germ line or somatic mutations of the SNF5 gene were detected in malignant rhabdoid tumors arising primarily in the kidney and brain. This gene has been shown to bind the c-myc protooncogene, as well as the BRCA1 and p53 tumor suppressors. Furthermore, SNF5 cooperates with HDAC (histone deacetylase) and loss of its function may thus inactivate HDAC. There is no evidence that silencing of these components of the SWI/SNF complex occurs by epigenetic means in EOC.

$B A F$. The mechanistic relationship between BAF complexes and the chromatin architecture inhibits target genes (21). BAF250 [also known as ARID1 (adenine-thymine rich interactive domain 1)] is responsible for directing the SWI/SNF complex to target promoters and regulates the transcription of certain genes by altering the chromatin structure around those genes (http://www.ncbi.nlm.nih.gov/gene/8289). ARID1 is also involved in the modulation of hormone-responsive promoters. The C-terminus of the protein is capable of stimulating glucocorticoid receptor-dependent transcriptional activation. Additionally, ARID1 is an essential gene for FAS (TNF receptor superfamily, member 6)-mediated apoptosis (22). ARID1A is one of the most frequently deleted genes across all cancer types $(23,24)$ and knockdown of this gene results in a failure of cell cycle arrest $(23,25)$. This alteration strongly predominates in kidney, breast and lung cancers in humans $(13,26)$. All inactivating mutations of this gene have been found in these cancers, suggesting that ARID1A plays an essential role during cancer development as a tumor suppressor.

More recent data from two groups have provided information about the profiles of CCC tumors with ARID1A inactivation (27,28). Inactivating mutations of ARID1A were identified in sporadic CCC samples and a variety of CCC cancer cell lines. ARID1A is considered to be the most commonly altered gene in EAOC, particularly in CCC, while alterations are rare in SAC. The mutation spectrum was enriched for $\mathrm{C}$ to $\mathrm{T}$ transitions at 5 '-CG base pairs. The

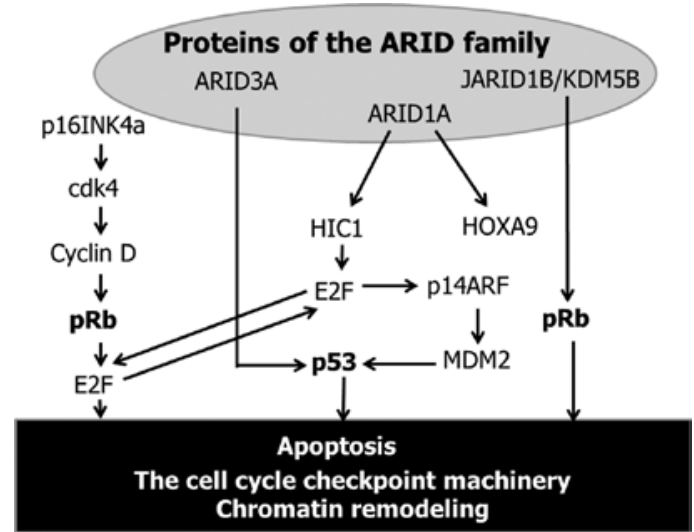

Figure 1. The role of ARID1A in the cell cycle checkpoint machinery. There are several ARID1A-interacting transcription factors; its downstream targets include HOXA9, HIC1, E2F, p53, pRb. Loss or dysfunction of the ARID1A gene may lead to aberrant chromatin remodeling, alterations in the cell cycle checkpoint machinery and subsequent apoptosis evasion.

mutations in ARID1A result in a stop codon or an out-offrame insertion or deletion. Atypical endometriotic lesions adjacent to the tumor (and not distant lesions) also exhibited the ARID1A mutations (27). Taken together, the data show that CCC may arise from atypical endometriosis with the mutation of ARID1A. Even in cases where genetic mutations of ARID1A have not been identified, other aberrations affecting its activity have been noted, such as abnormal promoter hypermethylation of ARID1A or aberrations of the downstream target genes, leading to low or normal protein levels, respectively. No conclusive evidence currently exists to support the existence of a role for ARID1A in tumor suppression in CCC.

Identification of ARID-containing proteins and ARIDIAinteracting components. A number of distinct human ARID proteins have been identified, including p270 (also known as ARID1A), KIAA1235 (ARID1B), RBP1 (retinol binding protein 1), RBP1L1 (ARID4B), RBP2 (retinol binding protein 2), SMCY/SMCX [KDM5D, lysine (K)-specific demethylase 5D], Plu-1 (KDM5B), jumonji (JARID2), Bright (DRIL1) (ARID3A), Bdp (DRIL-2) (ARID3B), MRF1 (ARID5A) and MRF2 (ARID5B) (29). Proteins of the ARID family are required for embryonic development and patterning, and maintain the expression pattern of homeotic genes at the chromatin level. As shown in Fig. 1, various BAF250a (ARID1A)-interacting proteins (transcription factor) exist, including HOXA9 and HIC1. ARID1A is a positive regulator of HOXA9 (30). HOXA9 expression is spatially and temporally regulated during hematopoiesis and embryonic development. Reduced HOXA9 transcript levels are reportedly associated with breast cancer aggression, metastasis and patient mortality (31). ARID1A also directly interacts with $\mathrm{HIC1}$ (hypermethylated in cancer 1), a tumor suppressor gene, which is epigenetically inactivated in numerous human cancers $(32,33)$. This gene encodes a transcriptional repressor involved in regulatory loops modulating p53-dependent and E2F-dependent cell survival and damage/ stress responses through the recruitment of ARID1A. E2F is a critical regulator of genes required for apoptosis by upregulating p53 and enhancing the p53-mediated activation of 
A

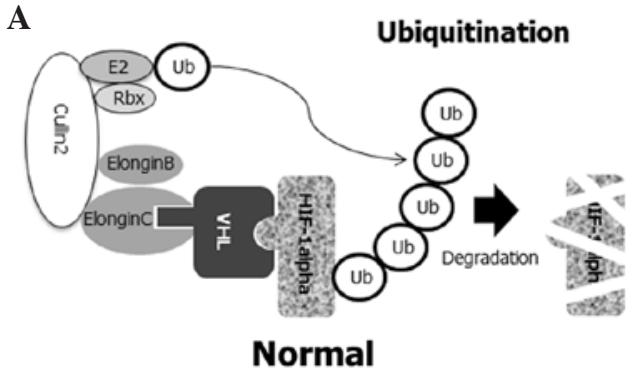

B

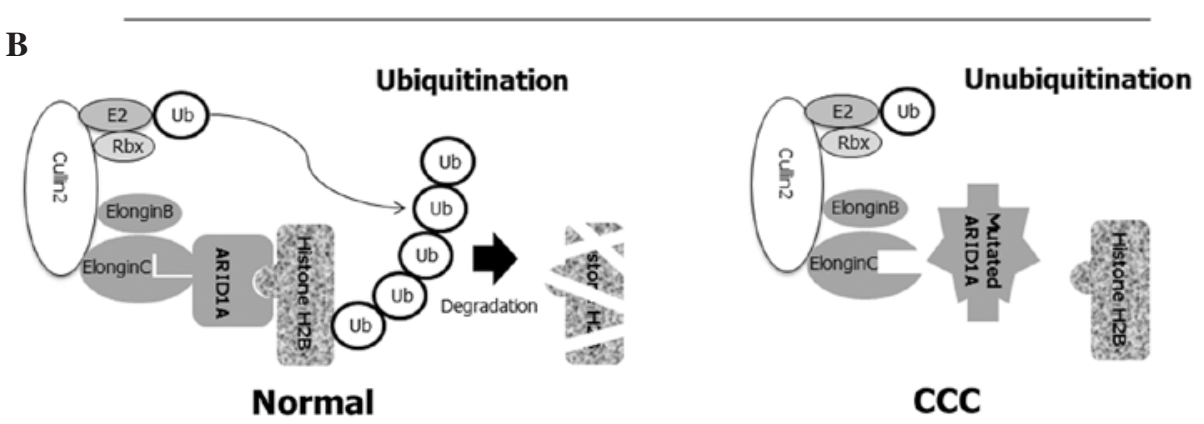

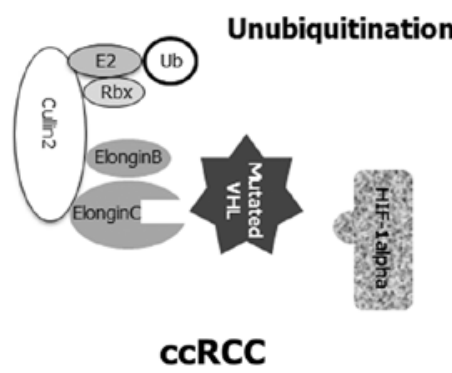

CCRCC

Figure 2. Putative molecular pathogenesis of CCC with ARID1A mutations. (A) The majority of ccRCC are caused by the mutation of the von Hippel-Lindau (VHL) tumor suppressor gene (54). VHL protein is part of an ubiquitin ligase complex that comprises elongin B, elongin C, cullin 2 and Rbx1, which are involved in ubiquitin-mediated destruction. This complex targets a hypoxia-inducible factor (HIF) transcription factor. In the absence of VHL, HIFresponsive genes and its downstream targets are activated. In ccRCC, when VHL protein either cannot function due to a mutation or is abnormally low/absent in the cell, HIF-1 $\alpha$ cannot be bound to the ubiquitin ligase, cannot be degraded, and thus is constitutively present at a higher level in the nucleus. High levels of HIF-1 $\alpha$ in turn lead to the overexpression of VEGF and other angiogenesis factors in carcinogenesis. (B) The molecular pathogenesis of ccRCC with VHL mutations resembles that noted in CCC patients with ARID1A mutations. ARID1A protein is also part of an ubiquitin ligase complex for ubiquitin-mediated destruction of histone H2B. Similar to the VHL gene, once histone H2B binds to ARID1A, an ubiquitin ligase complex binds H2B, leading to ubiquitination of $\mathrm{H} 2 \mathrm{~B}$, and marking it for degradation by the proteasomal machinery of the cell. The right panels of the figure show the disruption of this normal regulatory process when ARID1A function is aberrant by somatic mutations. In the absence of functional ARID1A, the ubiquitin ligase complex cannot bind H2B, resulting in the accumulation of H2B in the nucleus. Inactivation of the ARID1A tumor-suppressor protein and subsequent loss of function in the ARID1A complex result in dysfunction in the ubiquitination of histone $\mathrm{H} 2 \mathrm{~B}$, which is crucial in the aberration of chromatin remodeling and cell cycle checkpoint machinery, and subsequent evasion of apoptosis.

downstream pro-apoptotic genes (34). ARID3A is known as E2FBP1 (E2F binding protein-1; a protein that interacts with E2F) (35). ARID3A also interacts with p53 and is involved in the p53 regulatory pathway, suggesting that ARID3A plays a role in growth suppression mediated by p53 (35). In addition, p14ARF inhibits formation of the MDM2-p53 complex and subsequently prevents MDM2-induced p53 degradation (36). p14ARF stops cell growth at the G1/S and G2/M phases (36). Loss of the p53 signaling pathway occurs in human cancer either by p53 gene mutation (a direct mechanism) or by loss of cell signaling upstream and downstream of p53 (an indirect mechanism) in the remaining cancers expressing wild-type p53 gene (37).

Certain ARID family proteins are linked genetically with E2F-mediated transcriptional factor, which is regulated in part by $\mathrm{pRb}$, an active retinoblastoma tumor suppressor gene, resulting in cell cycle arrest (38). The p16INK4a-cdk4cyclin D-pRb pathway is modulated by ARID-dependent E2F signaling, resulting in cell cycle regulation (38). pRb phosphorylation leads to cell release from G1 arrest and to promotion entry into the S-phase (36). In addition, JARID1B/ KDM5B, a member of the ARID family, exerts cell cycle control via maintenance of $\mathrm{pRb}$ (39). These data allow us to speculate that proteins of the ARID family directly or indirectly regulate the cell cycle, particularly alterations in the cell cycle checkpoint machinery. In contrast to various other human tumor types, p53 and pRb mutations are only rarely detected in CCC. ARID1A mutations may be involved in the carcinogenesis of CCC through inactivation, but not mutation, of the cell cycle regulatory proteins such as p53 and pRb.

\section{A marked resemblance between $\mathrm{CCC}$ and ccRCC}

Clear cell cancers possess a marked similarity in gene expression profiles between CCC and ccRCC (40), suggesting that CCC and ccRCC are also pathogenetically similar. ccRCC is characterized by the presence of inactivating mutations in the VHL (von Hippel-Lindau) gene in the majority of cases (41). The VHL protein acts as the substrate recognition module of an E3 ubiquitin ligase complex by binding the substrate and adapters $(42,43)$. This protein directly interacts with Elongin C, whereas Elongin B connects VHL-Elongin C to cullin2-ROC1 (Rbx1) (Fig. 2A). These substrates include hypoxia-inducible factors (HIF-1 $\alpha$ and $-2 \alpha$ ). VHL forms a complex that retains the ability to ubiquitinate HIFs, resulting in degradation by targeting the hydroxylated HIF- $\alpha$ subunit for ubiquitination and proteasomal degradation. VHL protein acts as a master regulator of HIF-activity loss of the VHL protein due to germline or somatic mutations disrupting the formation of this complex, thereby reducing the ability of the 
VHC protein to ubiquitinate HIFs, resulting in accumulation of HIFs to high levels (42). Overexpression of HIF genes stimulates the expression of a number of significant genes, such as vascular endothelial growth factor (VEGF), plateletderived growth factor (PDGF) and transforming growth factor $\alpha(\mathrm{TGF} \alpha)(44)$.

As described above, the majority of CCC possess somatic inactivating mutations in the ARID1A gene $(27,28)$. As with VHL, ARID1A exists in complex with a series of other proteins, including elongin $\mathrm{B}$, elongin $\mathrm{C}$, cullin2 and $\mathrm{Rbx} 1$, to form an ubiquitin ligase complex (30) (Fig. 2B). Loss of the ARID1A tumor suppressor, which occurs with VHL mutations in ccRCC, promotes CCC tumorigenesis primarily through loss of the assembly of the Cullin-Elongin-Rbx1-E2 complex and subsequent ARID1A-mediated histone H2B regulation. The binding of $\mathrm{H} 2 \mathrm{~B}$ to ARID1 and to the ubiquitin ligase complex causes H2B to be ubiquitinated and degraded by the proteasomal complex (30). Mutated ARID1A may not bind to $\mathrm{H} 2 \mathrm{~B}$, and consequently is not degraded. Loss of the ARID1A protein may cause a failure to regulate the $\mathrm{H} 2 \mathrm{~B}$ assembly, resulting in accumulation of H2B to high levels. Results using siRNA-knockdown approach indicate that ARID1A is required for cell-cycle arrest (26). Ubiquitination of $\mathrm{H} 2 \mathrm{~B}$ was associated with transcriptional activation (45). For example, ubiquitinated H2B has been identified in the yeast Saccharomyces cerevisiae, and mutation of the conserved ubiquitination site is shown to confer defects in mitotic cell growth and meiosis. H2B ubiquitination is a prerequisite for a second modification on a different histone. Methylation of $\mathrm{H3}$ at lysine residues depends on the ubiquitination of histone H2B (46). Reduced ubiquitination of H2B reduces H3 lysine-79 di-methylation and correlates with a decreased gene expression. The addition of ubiquitin to chromatin components such as H2B affects various DNA-based processes, such as cell cycle progression, DNA damage repair and gene silencing (47). These results indicate the role of ARID1A mutations in components of the chromatin modification machinery in CCC.

Notably, mutations of the following three genes encoding enzymes involved in histone modification were found in ccRCC cases: UTX (KDM6A, a histone H3 lysine 27 demethy lase), SETD2 (a histone H3 lysine 36 methyltransferase), and JARID1C (KDM5C, a histone H3 lysine 4 demethylase) (48). KDM6A catalyzes the demethylation of tri/dimethylated histone $\mathrm{H} 3$ (48). This gene regulates cell proliferation and acts as a tumor suppressor via pRb-dependent pathways (49). SETD2 is a methyltransferase specific to histone $\mathrm{H} 3$ lysine36 , and methylation of this residue is associated with active chromatin. SETD2 behaves in the same manner as a potential tumor suppressor gene in breast cancer (50) and ccRCC (51). KDM5C encodes a protein with one ARID domain and is involved in the regulation of transcription and chromatin remodeling. These results emphasize the role of mutations in other components of the chromatin modification machinery in ccRCC (48).

Finally, the somatic mutation spectrum of the ARID1A gene in CCC tissue samples was calculated using the data from two recently published articles $(27,28)$. We calculated that the majority of these mutations were $\mathrm{C}$ to $\mathrm{T}$ mutations (68\%) (Table I). The mutation spectrum was enriched for tran-
Table I. Base substitution mutations of the ARID1A gene observed in CCC tissue samples.

\begin{tabular}{lrr}
\hline Base substitution mutations & No. & $(\%)$ \\
\hline Transition total & 21 & $(75.0)$ \\
C to T & 19 & $(67.9)$ \\
A to G & 1 & $(3.6)$ \\
G to A & 1 & $(3.6)$ \\
Transversion total & 7 & $(25.0)$ \\
C to A & 1 & $(3.6)$ \\
C to G & 1 & $(3.6)$ \\
A to C & 1 & $(3.6)$ \\
G to T & 2 & $(7.1)$ \\
T to A & 1 & $(3.6)$ \\
T to G & 1 & $(3.6)$
\end{tabular}

The majority of base substitution mutations were $\mathrm{C}$ to $\mathrm{T}$ transition. Data are from refs. 27 and 28.

sitions (75\%), indicating that $\mathrm{C}$ to $\mathrm{T}$ transition mutations were the major type of base substitution. Notably, the mutation spectrum in ccRCC was dominated by $\mathrm{C}$ to $\mathrm{T} / \mathrm{G}$ to $\mathrm{A}$ transitions, but not transversion (48). The mutation spectrum in CCC was dominated by $\mathrm{C}$ to $\mathrm{T}$ transition as has been noted in ccRCC. These data allow us to hypothesize that CCC and ccRCC arise from a common pathogenesis. The data on the ccRCC gene mutations may provide insights into pathogenesis as well as the opportunity to gain a better understanding of the role of genetic and epigenetic subtypes of CCC. VHL inactivation alone induces senescence, suggesting a requirement for additional mutations to further drive ccRCC development in VHL mutant cases (48). No data exist regarding whether ARID1A inactivation alone induces CCC phenotype. Therefore, the role of ARID1A and the manner in which its inactivation affects cancer development should be investigated.

\section{Conclusions}

Although the broad principles of the biology of CCC are not fully understood, this tumor type is often associated with endometriosis. The somatic mutations of $\mathrm{CCC}$ have been investigated. Although p53, KRAS and PTEN genes are frequently mutated in other types of human cancer, contribution of these genes to neoplastic transformation of CCC is limited $(27,52)$. One of the novel mutated genes involved in CCC was found to be ARID1A, a chromatin remodeling modifier $(27,28)$. This review focuses on the potential role of histone modifiers such as ARID1A in CCC carcinogenesis. The mutation spectrum of ARID1A in CCC was dominated by $\mathrm{C}$ to $\mathrm{T}$ transitions, leading to nonsense mutations $(27,28)$. Since ARID1A mutation and subsequent loss or dysfunction of BAF250a protein is observed in preneoplastic lesions (atypical endometriosis), it has been reported that this mutation is an early event in the transformation of endometriosis into CCC (27). The biological role of ARID1A is to ubiquitinate 
and rapidly degrade histone $\mathrm{H} 2 \mathrm{~B}$ through the proteosomal complex (30). This process includes the pathway from aberrations in ARID1A to dysregulated (unubiquitinated) histone $\mathrm{H} 2 \mathrm{~B}$, leading to downstream changes in transcription genes. Post-translational modifications of the histone $\mathrm{H} 2 \mathrm{~B}$ are centrally involved in the regulation of all DNA-templated processes, including gene transcription, DNA replication, recombination, and repair, thus regulating a wide range of cellular processes and functions.

Histone modifications and other epigenetic mechanisms work together in maintaining gene activity states. Epigenetic information in chromatin includes covalent modifications, such as acetylation, methylation, phosphorylation and ubiquitination, of histones. Therefore, ARID1A, a chromatinmodifying factor, plays an essential role in DNA processing pathways that dictate cellular functions. ARID1A may contribute to cell cycle arrest and induced apoptosis, possibly through p53- and/or pRb-dependent signaling cascades, suggesting a role of ARID1A in the cell cycle checkpoint machinery (Fig. 2). Loss or dysfunction of the ARID1A gene by mutations may lead to aberrant chromatin remodeling, alterations in the cell cycle checkpoint machinery and subsequent apoptosis evasion. ARID1A acts as a tumor suppressor gene, that stimulates cell signaling, leading to cell cycle arrest and cell death in the event of DNA damage. Therefore, the dysfunction of ARID1A may lead to susceptibility to CCC carcinogenesis through a defect in the repair or replication of damaged DNA. Loss of expression and dysfunction of the ARID family may markedly alter in carcinogenesis. However, the functions and regulation of histone $\mathrm{H} 2 \mathrm{~B}$ ubiquitination and deubiquitination by ARID1A have yet to be fully understood. This is a significant consideration for various tumors showing the downregulation of ARID1A expression since acquired resistance to the activation of apoptosis in tumor cells is a serious limitation of current anticancer therapies. Thus investigating the manner in which ARID1A levels in $\mathrm{CCC}$ affect the prognosis of the disease upon treatment with various DNA-damaging agents is crucial.

Although the broad principles of the biology of CCC are not fully understood, significant advances have been made. Recent biochemical studies based on genome-wide expression analysis technology have noted a specific expression of a transcription factor, hepatocyte nuclear factor-1 $\beta$ (HNF-1 $\beta$ ), in $\mathrm{CCC}(8,53)$. HNF-1 $\beta$ overexpression is common in CCC. By comparing $\mathrm{CCC}$ to the contiguous atypical endometriotic lesions, the same overexpression of HNF-1 $\beta$ may be present in the putative precursor lesions (atypical endometriosis) and in a variety cancers. The distant endometriotic lesions have also shown a moderate expression of HNF-1 $\beta$. In the case of EAC, however, HNF-1 $\beta$ expression was not present in the atypical endometriosis nor in the tumors. A correlation between chromatin remodeling gene ARID1A and the CCC-specific gene HNF-1 $\beta$ has yet to be elucidated.

Although ARID1A mutation may be significant factor for CCC carcinogenesis, various questions have yet to be answered. Investigation into whether i) histone modification is the most critical event for oncogenesis; ii) other, as yet understudied, genes are also involved; and iii) these events are more critical for CCC and not in another organ system, is required to determine new treatment modalities for CCC.

\section{Acknowledgements}

This review was supported by KAKENHI (Japan Society for the Promotion of Science (JSPS) Grant-in-Aid). We thank all the study participants for their time and efforts. We thank Mr. Mikiko Kita for editorial assistance.

\section{References}

1. Piver MS: Prophylactic oophorectomy: reducing the U.S. death rate from epithelial ovarian cancer. A continuing debate. Oncologist 1: 326-330, 1996.

2. Kennedy AW, Biscotti CV, Hart WR and Webster KD: Ovarian clear cell adenocarcinoma. Gynecol Oncol 32: 342-349, 1998.

3. Ryu SY, Park SI, Nam BH, et al: Prognostic significance of histological grade in clear-cell carcinoma of the ovary: a retrospective study of Korean Gynecologic Oncology Group. Ann Oncol 20: 1032-1036, 2009.

4. Viganó P, Somigliana E, Chiodo I, Abbiati A and Vercellini P: Molecular mechanisms and biological plausibility underlying the malignant transformation of endometriosis: a critical analysis. Hum Reprod Update 12: 77-89, 2006.

5. Kobayashi H, Sumimoto K, Moniwa N, et al: Risk of developing ovarian cancer among women with ovarian endometrioma: a cohort study in Shizuoka, Japan. Int J Gynecol Cancer 17: 37-43, 2007.

6. Bell DA: Origins and molecular pathology of ovarian cancer. Mod Pathol 18 (Suppl 2): S19-S32, 2005.

7. Jiang X, Hitchcock A, Bryan EJ, et al: Microsatellite analysis of endometriosis reveals loss of heterozygosity at candidate ovarian tumor suppressor gene loci. Cancer Res 56: 3534-3539, 1996.

8. Kobayashi H, Kajiwara H, Kanayama S, et al: Molecular pathogenesis of endometriosis-associated clear cell carcinoma of the ovary (Review). Oncol Rep 22: 233-240, 2009.

9. Yoo AS and Crabtree GR: ATP-dependent chromatin remodeling in neural development. Curr Opin Neurobiol 19: 120-126, 2009.

10. Weissman B and Knudsen KE: Hijacking the chromatin remodeling machinery: impact of SWI/SNF perturbations in cancer. Cancer Res 69: 8223-8230, 2009.

11. Osipovich OA, Subrahmanyam R, Pierce S, Sen R and Oltz EM: Cutting edge: SWI/SNF mediates antisense Igh transcription and locus-wide accessibility in B cell precursors. J Immunol 183: 1509-1513, 2009.

12. Kundu S and Peterson CL: Role of chromatin states in transcriptional memory. Biochim Biophys Acta 1790: 445-455, 2009.

13. Rodriguez-Nieto S and Sanchez-Cespedes M: BRG1 and LKB1: tales of two tumor suppressor genes on chromosome 19p and lung cancer. Carcinogenesis 30: 547-554. 2009.

14. Abbas T and Dutta A: p21 in cancer: intricate networks and multiple activities. Nat Rev Cancer 9: 400-414, 2009.

15. Toyota $\mathrm{M}$ and Suzuki H: Epigenetic drivers of genetic alterations. Adv Genet 70: 309-323, 2010.

16. Fan D, Ma C and Zhang H: The molecular mechanisms that underlie the tumor suppressor function of LKB1. Acta Biochim Biophys Sin (Shanghai) 41: 97-107, 2009.

17. Hess J, Angel P and Schorpp-Kistner M: AP-1 subunits: quarrel and harmony among siblings. J Cell Sci 117: 5965-5973, 2004.

18. Hydbring P and Larsson LG: Cdk2: a key regulator of the senescence control function of Myc. Aging 2: 244-250, 2010.

19. Bogliolo M, Cabré O, Callén E, et al: The Fanconi anaemia genome stability and tumour suppressor network. Mutagenesis 17: 529-538, 2002.

20. Sansam CG and Roberts CW: Epigenetics and cancer: altered chromatin remodeling via Snf5 loss leads to aberrant cell cycle regulation. Cell Cycle 5: 621-624, 2006.

21. Watanabe H, Mizutani T, Haraguchi T, et al: SWI/SNF complex is essential for NRSF-mediated suppression of neuronal genes in human nonsmall cell lung carcinoma cell lines. Oncogene 25: 470-479, 2006

22. Luo B, Cheung HW, Subramanian A, et al: Highly parallel identification of essential genes in cancer cells. Proc Natl Acad Sci USA 105: 20380-20385, 2008.

23. Crow E, Du Z and Li L: New insights into prion biology from the novel $\left[\mathrm{SWI}^{+}\right]$system. Prion 2: 141-144, 2008.

24. Mertens F, Johansson B, Höglund $M$ and Mitelman F: Chromosomal imbalance maps of malignant solid tumors: a cytogenetic survey of 3185 neoplasms. Cancer Res 57: 2765-2780, 1997. 
25. Nagl NG Jr, Patsialou A, Haines DS, et al: The p270 (ARID1A/ SMARCF1) subunit of mammalian SWI/SNF-related complexes is essential for normal cell cycle arrest. Cancer Res 65: 9236-9244, 2005.

26. Wang X, Nagl NG Jr, Flowers S, et al: Expression of p270 (ARID1A), a component of human SWI/SNF complexes, in human tumors. Int J Cancer 112: 636, 2004.

27. Wiegand KC, Shah SP, Al-Agha OM, et al: ARID1A mutations in endometriosis-associated ovarian carcinomas. $\mathrm{N}$ Engl J Med 363: 1532-1543, 2010.

28. Jones S, Wang TL, Shih IeM, et al: Frequent mutations of chromatin remodeling gene ARID1A in ovarian clear cel carcinoma. Science 330: 228-231, 2010

29. Wilsker D, Patsialou A, Dallas PB and Moran E: ARID proteins: a diverse family of DNA binding proteins implicated in the control of cell growth, differentiation, and development. Cell Growth Differ 13: 95-106, 2002.

30. Li XS, Trojer P, Matsumura T, Treisman JE and Tanese N: Mammalian SWI/SNF - a subunit BAF250/ARID1 is an E3 ubiquitin ligase that targets histone H2B. Mol Cell Biol 30: $1673-1688,2010$

31. Gilbert PM, Mouw JK, Unger MA, et al: HOXA9 regulates BRCA1 expression to modulate human breast tumor phenotype. J Clin Invest 120: 1535-1550, 2010.

32. Dehennaut V and Leprince D: Implication of HIC1 (hypermethylated in cancer 1) in the DNA damage response. Bull Cancer 96: E66-E72, 2009.

33. Van Rechem C, Boulay G and Leprince D: HIC1 interacts with a specific subunit of SWI/SNF complexes, ARID1A/BAF250A. Biochem Biophys Res Commun 385: 586-590, 2009.

34. Iaquinta PJ and Lees JA: Life and death decisions by the E2F transcription factors. Curr Opin Cell Biol 19: 649-657, 2007.

35. Ma K, Araki K, Ichwan SJ, et al: E2FBP1/DRIL1, an AT-rich interaction domain-family transcription factor, is regulated by p53. Mol Cancer Res 1: 438-444, 2003.

36. Kim YT and Zhao M: Aberrant cell cycle regulation in cervical carcinoma. Yonsei Med J 46: 597-613, 2005.

37. Bourdon JC: p53 and its isoforms in cancer. Br J Cancer 97: 277-282, 2007

38. Staehling-Hampton K, Ciampa PJ, Brook A and Dyson N: A genetic screen for modifiers of E2F in Drosophila melanogaster. Genetics 153: 275-287, 1999.

39. Roesch A, Mueller AM, Stempfl T, et al: RBP2-H1/JARID1B is a transcriptional regulator with a tumor suppressive potential in melanoma cells. Int J Cancer 122: 1047-1057, 2008
40. Zorn KK, Bonome T, Gangi L, et al: Gene expression profiles of serous, endometrioid, and clear cell subtypes of ovarian and endometrial cancer. Clin Cancer Res 11: 6422-6430, 2005.

41. Gnarra JR, Tory K, Weng Y, et al: Mutations of the VHL tumour suppressor gene in renal carcinoma. Nat Genet 7: 85-90, 1994.

42. Hacker KE, Lee CM and Rathmell WK: VHL type 2B mutations retain VBC complex form and function. PLoS One 3: E3801, 2008.

43. Vodermaier HC: APC/C and SCF: controlling each other and the cell cycle. Curr Biol 14: R787-R796, 2004.

44. Clark PE: The role of VHL in clear-cell renal cell carcinoma and its relation to targeted therapy. Kidney Int 76: 939-945, 2009.

45. Beisel C, Imhof A, Greene J, Kremmer E and Sauer F: Histone methylation by the Drosophila epigenetic transcriptional regulator Ash1. Nature 419: 857-862, 2002.

46. Ezhkova E and Tansey WP: Proteasomal ATPases link ubiquitylation of histone $\mathrm{H} 2 \mathrm{~B}$ to methylation of histone H3. Mol Cell 13 435-442, 2004

47. Atanassov BS, Koutelou E and Dent SY: The role of deubiquitinating enzymes in chromatin regulation. FEBS Lett: Oct 26 , 2010 (Epub ahead of print)

48. Dalgliesh GL, Furge K, Greenman C, et al: Systematic sequencing of renal carcinoma reveals inactivation of histone modifying genes. Nature 463: 360-363, 2010.

49. Wang JK, Tsai MC, Poulin G, et al: The histone demethylase UTX enables RB-dependent cell fate control. Genes Dev 24: 327-332, 2010.

50. Newbold RF and Mokbel K: Evidence for a tumour suppressor function of SETD2 in human breast cancer: a new hypothesis. Anticancer Res 30: 3309-3311, 2010.

51. Duns G, van den Berg E, van Duivenbode I, et al: Histone methyltransferase gene SETD2 is a novel tumor suppressor gene in clear cell renal cell carcinoma. Cancer Res 70: 4287-4291, 2010.

52. Kurman RJ and Shih IeM: Pathogenesis of ovarian cancer: lessons from morphology and molecular biology and their clinical implications. Int J Gynecol Pathol 27: 151-160, 2008.

53. Kajihara H, Yamada Y, Kanayama S, et al: Clear cell carcinoma of the ovary: Potential pathogenic mechanisms (Review). Oncol Rep 23: 1193-1203, 2010.

54. Sufan RI, Jewett MA and Ohh M: The role of von HippelLindau tumor suppressor protein and hypoxia in renal clear cell carcinoma. Am J Physiol Renal Physiol 287: F1-F6, 2004 